\title{
Dynamics of Liquid-Liquid Mixing: A 2-Zone Model
}

\author{
JOS M. H. JANSSEN* and HAN E. H. MEIJER \\ Center for Polymers and Composites \\ Eindhoven University of Technology \\ 5600 MB Eindhoven, The Netherlands
}

\begin{abstract}
The development of multiphase liquid-liquid morphologies during mixing at small Reynolds numbers has been modeled. The mixing process is divided into i) stretching of dispersed drops, ii) breakup of the liquid threads formed, and iii) coalescence of the final droplets upon collision. Rules and criteria of the distinct processes are presented and combined to a general 2-zone mixing model simplifying the flow field into a sequence of alternating "strong and weak zones." In a "strong zone," dispersed drops and threads are stretched unless their radius is too small; meanwhile, the stretching threads might break up into droplets. In the subsequent "weak zone," the remaining threads may disintegrate while any drops present may coalesce. After passing a number of zones, stretching, breakup, and coalescence lead to a dynamic equilibrium that could be considered as the "final" morphology. Using the 2-zone mixing model, the influence of material parameters and processing conditions on the morphology has been studied. Interestingly, increasing either viscosity (dispersed or continuous phase) yields a finer morphology due to the delay of thread breakup (allowing for further stretching) and suppression of coalescence.
\end{abstract}

\section{INTRODUCTION}

\section{Blend Morphologies}

1 specific properties that are superior to those of the constituents. Since most polymers are immiscible in the molten state, the compounding process yields a heterogeneous morphology that is characterized by the shape, size, and distribution of the constituting domains. Since the properties of a blend are largely influenced by its morphology, it is essential to model the development of the morphology during compounding. Any mixing model should incorporate the governing mechanisms with their principally transient characteristics: the time scales of the distinct micro-rheological processes have to be considered to see whether the available process time suffices for a specific event to occur. A frozen-in morphology is not necessarily in its equilibrium state and, in a subsequent processing step the morphology achieved may change as a result of the processing conditions there.

Fundamental research on the mixing of liquidliquid systems often focuses on idealized systems: isolated drops of well-characterized model liquids in

*Present address, for correspondence: General Elcctric Plastics, P.O. Box 117,4600 AC Bergen op Zoom, The Netherlands. elementary flows. Results are generally given in dimensionless representation suitable for scaling to practical processes. Most of the fundamental studies are restricted to Newtonian (viscous) systems, although viscoelasticity is an essential feature of polymeric melts and solutions. The question arises whether the influence of viscoelasticity is such that it should necessarily be taken into account in the distinct mixing mechanisms. An analysis of its relevance might result in an approval of the desirable omission of viscoelasticity that was, up to now, mostly caused by the complexity of the problem.

\section{Mixing Mechanisms}

In the mixing of immiscible liquids, the minor component is generally (but not necessarily) present as the dispersed phase (drops or filaments) in a continuous phase of the major component. An elementary step in the mixing process is the deformation of a dispersed drop in a flow field. For molten polymers, the Reynolds number is small so that inertia can be neglected with respect to viscous shear stresses. Furthermore, the density difference and hence the Bond number is small, implying that buoyancy effects are negligible with respect to interfacial effects. Drop deformation is then mainly governed by the capillary number, the ratio of the (deforming) shear stress $\tau$ exerted on the drop by the external flow field, and the 
(shape conserving) interfacial stress $\sigma / R$ (with $\sigma$ the interfacial tension and $R$ the radius):

$$
C a=\frac{\tau R}{\sigma}=\frac{\eta_{c} \dot{\gamma} R}{\sigma}
$$

with $\eta_{c}$ the viscosity of the continuous phase and $\dot{\gamma}$ the shear rate, defined as:

$$
\dot{\gamma}=\sqrt{2 \mathbf{D}: \mathbf{D}}
$$

D being the rate of deformation tensor. (Note that in 2-D and 3-D elongational flow $\dot{\gamma}$ equals $2 \dot{\varepsilon}$ and $\dot{\varepsilon} \sqrt{3}$ respectively, with $\dot{\varepsilon}$ being the elongation rate.) If the capillary number is small, the interfacial stress withstands the shear stress and a steady ellipsoid drop shape exists. Above a critical value, $\mathrm{Ca}_{\text {crit }}$, the viscous shear stress overrules the interfacial stress and no stable equilibrium drop shape exists. The drop is stretched and finally breaks into fragments.

Experimentally, $C \mathrm{C}_{\text {crit }}$ is determined by slowly increasing the shear rate and evaluating whether or not the deformed drop shape is stable on the long run; in fact, this is drop breakup under quasiequilibrium conditions. Taylor (1) and Grace (2) showed that the critical capillary number depends both on the flow type and the viscosity ratio $p$ between dispersed and continuous phase:

$$
p=\eta_{\mathrm{d}} / \eta_{\mathrm{c}}
$$

From Fig. 1 it can be seen that under quasi-equilibrium conditions it is easiest to deform and break drops around $p \sim 1$. Furthermore, elongational flow is more effective than simple shear flow. If $p>4$ it is impossible to break drops in simple shear because of the rotational character of the flow. Numerous investigators confirmed Fig. 1 either experimentally or numerically (e.g., 3, 4).

A useful subdivision of the mixing process can be based on the value of the (local) capillary number,

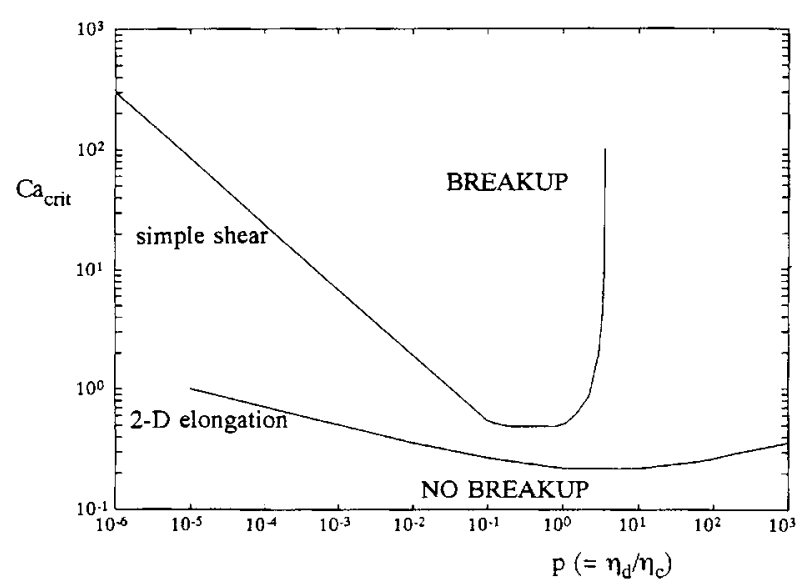

Fig. 1. Critical capillary number for drop breakup vs. viscosity ratio $p$ in simple shear and 2-D (plane) elongational flow; after (2). which continuously decreases during the process as a result of the length scale reduction (roughly from $1 \mathrm{~mm}$ to $1 \mu \mathrm{m}$ ):

i) Stretching of dispersed drops when $\mathrm{Ca} \gg \mathrm{Ca}_{\text {crit }}$ : Typically in the initial stage of mixing, the dispersed domains are large and liquid threads are formed.

ii) Breakup of these threads when $\mathrm{Ca} \sim 1$ : The interfacial stress $\sigma / R$ becomes active and favors breakup. Depending on their size, the drops formed may break again.

iii) Coalescence of the dispersed droplets when $\mathrm{Ca} \ll$ 1: Coalescence preferentially takes place with small drops and in quiet regions of the mixer, as is shown below.

The aim of this work is to discuss the dynamics of the distinct mechanisms (see also 5,6 ) and to combine them into an overall 2 -zone mixing model that simulates the evolution of a morphology during compounding.

\section{STRETCHING}

\section{Affine Deformation}

As a typical starting morphology, a drop-in-matrix structure is considered with the drop size of the order of magnitude of a millimeter. In polymer processing, some attention has been given to lamellar starting morphologies originating from the melting stage $(7,8)$. These lamellae break up into threads and subsequently into drops. In a more general approach of the mixing process, such a melting stage is omitted and a large initial length scale is considered as the starting point. For immiscible polymer melts, an order of magnitude estimate (e.g., $\eta_{c} \sim 10^{2} \mathrm{~Pa} \cdot \mathrm{s}, \dot{\gamma} \sim 10^{2}$ $\mathrm{s}^{-1}, \sigma \sim 10^{-2} \mathrm{~N} / \mathrm{m}$, and $R \sim 10^{3} \mathrm{~m}$ ) yields the capillary number $\mathrm{Ca} \sim 10^{3}$ so that $\mathrm{Ca} \gg \mathrm{Ca}_{\text {crit }}$. In other words, in the initial stage the shape conserving interfacial stress $(\sigma / R)$ is overruled by the deforming shear stress, and the drops are deformed affinely with the matrix: stretching with passive interfaces or distributive mixing. Note that for miscible liquids (no interfacial tension) this convective mixing is the only relevant process (apart from some interdiffusion).

In the case of affine motion, the stretching of a drop (with radius $R_{0}$ and "length" $L_{0}=2 R_{0}$ ) into a thread (with radius $R$ and length $L$ ) can easily be calculated from the global flow field. In elongational flow, stretching proceeds exponentially:

$$
L / L_{\mathrm{O}}=e^{\varepsilon}, \quad R / R_{\mathrm{O}}=e^{-\varepsilon / 2}
$$

where it is assumed that the cross-section remains circular, which is the case in 3-D (or uniaxial) elongation but not in 2-D (plane) elongation, where the extending thread would, in fact, flatten. However, in general, the interfacial tension guarantees a circular cross section. In simple shear flow, affine deformation proceeds slower: The long axis of the extending drop 
turns away from the principal direction of strain rate $\left(45^{\circ}\right)$ and the length grows approximately linearly:

$$
\begin{gathered}
L / L_{0}=\frac{1}{2} \gamma+\frac{1}{2} \sqrt{4+\gamma^{2}} \approx \gamma \\
R / R_{0}=\left(\frac{1}{2} \gamma+\frac{1}{2} \sqrt{4+\gamma^{2}}\right)^{-1 / 2} \approx \gamma^{-1 / 2}
\end{gathered}
$$

where the approximations only hold for large shear values. Evidently, affine stretching depends only on the total deformation applied ( $\varepsilon$ or $\gamma$ ); deformation rate and time are interchangeable as long as $\mathrm{Ca} \gg$ $C a_{\text {crit }}$. It was determined experimentally that an excess of $\mathrm{Ca} / \mathrm{Ca}$ crit $>5$ is sufficient to neglect the interfacial stress $\sigma / R$ and apply the simple rules of affine deformation $(6,9)$. Note that in simple shear, affine deformation is possible only for viscosity ratios $p<4$ since for larger $p, C a_{\text {crit }} \rightarrow \infty$. In a practical complex flow field, however, a drop once deformed sufficiently (no matter how) always continues stretching affinely, even if $p>4$ and simple shear prevails.

\section{Stretching and Folding}

In simple shear flow (at constant $\dot{\gamma}$ ), affine deformation proceeds only linearly instead of exponentially as in elongational flows (at constant $\dot{\varepsilon}$ ), which originates from the difference in the rotational contribution. Unfortunately, most practical flows are dominated by simple shear, since elongation at constant stretching rate is difficult to sustain for long times. However, by repeated reorientation of an extending drop in simple shear, directing its main axis perpen- dicular to the streamlines, the stretching efficiency can be enhanced: the drop axis passes through the $45^{\circ}$ direction again and again. Moreover, the efficiency of mixing can be enhanced by a combination of stretching and folding, the so-called "baker's transformation" $(10,11)$ that changes the efficiency of the distributive mixing from a linear dependence on the strain applied to an exponential dependence. The principle is depicted schematically in Fig. 2. Every fold doubles the number of filaments to be stretched simultaneously. The quality of mixing, characterized by either the total interfacial area, the number of layers, or the reciprocal of the striation thickness, increases exponentially.

Application of the baker's transformation is realized in most well-designed static mixers (e.g., Ross ISG, Multiflux, Kenics, Sulzer SMX, as discussed in Ref. 5). A beautiful visualization of stretching and folding in a continuous flow is obtained in the socalled "cavity flow" $(12,13)$. The cavity is a rectangular cell (quasi 2-D) filled with, e.g., glycerin. The two short walls are static while the two long walls can translate independently as moving belts. The deformation of an injected tracer line or drop (passive: colored glycerine; without interfacial tension) is studied in dependence on the protocol of subsequent wall movements. In Fig. 3, the numerical simulations of some typical experiments are presented. [These simulations were based on the analytical solution of the 2-D Stokes flow problem $(14,15)$.] In all three cases the initial condition is a straight vertical line through the center of the cell while the total wall displacement applied equals 25 times the length of the top wall. In the first example only the top wall moves: mixing

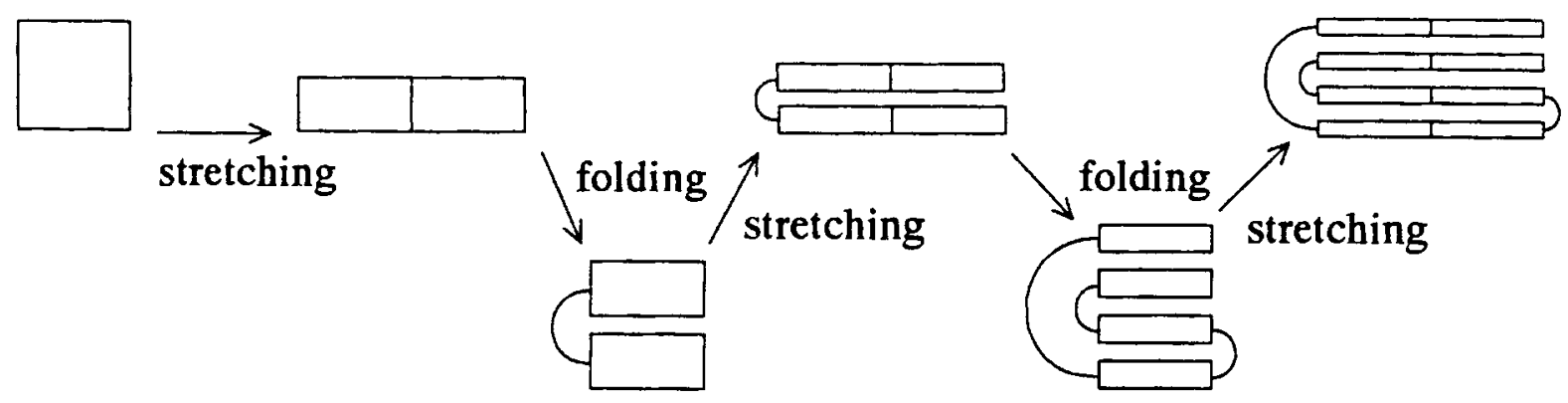

Fig. 2. The baker's transformation: stretching and folding, resulting in exponential mixing.
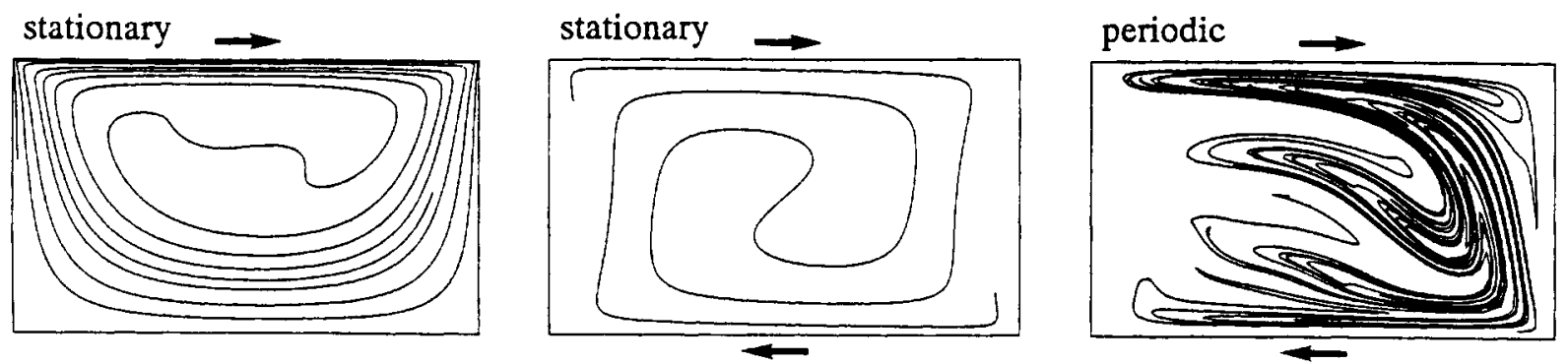

Fig. 3. Deformation of a passive tracer line in a cavity: i) the top wall moves stationary; ii) top and bottom wall move stationary: iii) top and bottom wall move periodically one after the other for five periods. The total wall displacement is 25 times the top wall length in all three cases. 
proceeds linearly. In the second example both top and bottom wall move simultaneously at the same speed with a displacement of 12.5 times the top wall length for each wall: the flow pattern is close to a rigid body rotation and the tracer line is stretched less than in the former case. In the last example, again top and bottom wall move in clockwise direction but now in a periodic way, i.e., subsequently, one after the other for five periods in total with a wall displacement of 2.5 top wall lengths during each half a period: folds emerge, indicating deterministic chaos with exponential mixing. The reason for the appearance of folds is the fact that the streamline pattern (closely resembled in the first flow example) flips upside down after each half period, yielding "crossing streamlines" in time: locally, the flow direction changes and the stretching line is folded. Analyzing the periodic flow pattern in the cavity, chaotic regions are found, but also "islands" that travel around the cavity without being stretched. In practical situations the aim should be to minimize such unmixed islands.

Apart from static mixers and textbook examples like the cavity flow, also practical dynamic mixers, e.g., extruders, may approach the principles of reorienting and folding. An example is the intermeshing co-rotating twin-screw extruder. During the takeover of the material from one screw to the other, folds and reorientations are (partially) achieved. In an intermeshing counter-rotating twin-screw extruder, however, effectively closed volumina of material are transported, which is not very efficient for distributive mixing. Single-screw extruders are inferior for distributive mixing, since no reorientations or folds are introduced. Only special types like the Buss Co-kneader (16) can achieve efficient distributive mixing. In this particular design an axial oscillation is superimposed to the tangential rotation, while pins are inserted into the screw channel. As a result the material is woven and folded in a way that is very similar to the operating principle of an ordinary eggbeater.

\section{BREAKUP}

\section{Thread Breakup}

When the original drops are stretched into long, slender filaments, local radii are decreased such that the interfacial tension starts playing a role (active interfaces). Following the order of magnitude estimation of the former section, it can be seen that the shear stress $\tau$ and the interfacial stress $\sigma / R$ become of the same order of magnitude $(\mathrm{Ca} \sim 1)$ if the radius of the threads has decreased to $1 \mu \mathrm{m}$. The interfacial tension tends to minimize the interfacial area between the two phases. As a consequence, small disturbances present at the interface of the liquid cylinder grow and finally result in the disintegration of the thread into a line of drops.

For viscous systems, sinusoidal disturbances grow exponentially in time. The breakup time of a thread (with radius $R_{0}$ ) in a quiescent matrix is given by
$(17,18)$ :

$$
t_{b}=\frac{2 \eta_{c} R_{0}}{\sigma \Omega_{m}} \ln \left(\frac{\alpha_{b}}{\alpha_{0}}\right)
$$

where $\alpha_{b}$ is the disturbance amplitude at breakup $\left(\alpha_{b} \approx 0.82 R_{0}\right)$ and $\alpha_{0}$ the initial amplitude. A lower limit of $\alpha_{0}$ is given by thermal fluctuations and was estimated by Kuhn (19): $\alpha_{0}=\left(21 k T /\left(8 \pi^{3 / 2} \sigma\right)\right)^{1 / 2} \sim$ $10^{-9} \mathrm{~m}$ with $k$ the Boltzmann constant and $T$ the absolute temperature. In practice, even at volume fractions $\phi$ as low as $1 \%$, neighboring particles disturb the flow field locally and hence impose interfacial disturbances that may be much larger than Kuhn's estimate (see, e.g., Fig. 6). However, the influence of $\phi$ and $\mathrm{Ca}$ (i.e., the deformability of the interface) on $\alpha_{0}$ is not yet modeled adequately.

$\Omega_{m}$ is the dimensionless growth rate of the dominant disturbance and depends on the viscosity ratio $p$; see Fig. 4. The size of the drops formed upon breakup is obtained from conservation of volume:

$$
R_{\mathrm{drops}}=R_{\mathrm{O}} \sqrt[3]{\frac{3 \pi}{2 x_{m}}}
$$

with $x_{m}$ the dominant wavenumber $\left(x=2 \pi R_{0} / \lambda\right)$, also given in Fig. 4. For instance, if the viscosity ratio $p=1, x_{m}=0.56$ so that the droplets formed will be twice as thick as the original thread. The disturbance growth rate $\Omega_{m}$ in fact only holds for small amplitudes. However, calculations with the finite element method showed that only a slight error is introduced upon extrapolation to large amplitudes (6).

Stretching of a liquid thread has a stabilizing effect against breakup (20-23): The thinning thread sustains stable until it has reached a specific radius $R_{\text {crit }}$. Meanwhile, it is stretched affinely ( $E q 4$ ), and interfacial disturbances of different wavenumbers grow and damp without leading to breakup. Then, at $R_{\text {crit }}$ the fatal disturbance starts growing and after the growth time $t_{g}$ disintegration takes place into

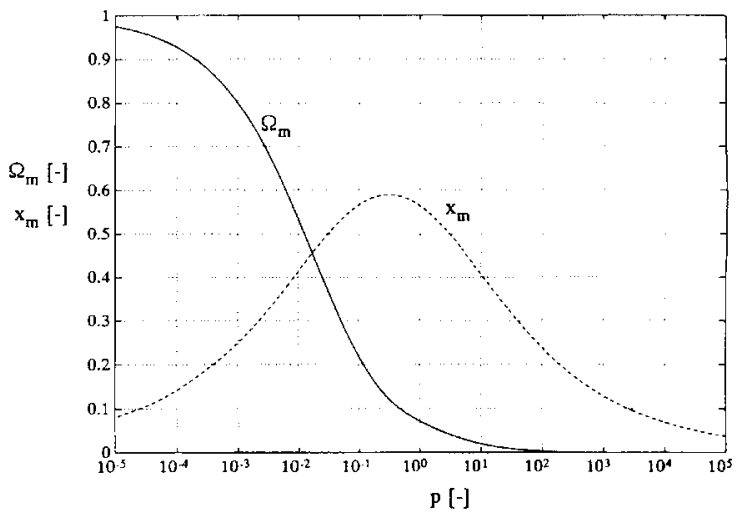

Fig. 4. Dominant wave number $x_{m}$ and corresponding growth rate $\Omega_{m}$ of interfacial disturbances us. the viscosity ratio $p$ $\left(=\eta_{d} / \eta_{c}\right):$ after $(17)$ 
drops of radius $R_{\text {drops }}$. For the case of a constant stretching rate $\dot{\varepsilon}$, the value of $R_{\text {crit }}$ is given in Fig. 5; it depends only on the flow parameter $\eta C \dot{\varepsilon} / \sigma$ and the viscosity ratio, not on the initial radius of the thread. Generally, the higher any of the viscosities $\eta_{d . c}$, the stronger the stabilizing effect and thus the thinner the thread at the moment of breakup. The resulting drop size $R_{\text {drops }}$ can be approximated well from $R_{\text {crit }}$ since the wavenumber at breakup is almost constant:

$$
R_{\mathrm{drops}} \approx 1.7 R_{\mathrm{cril}}
$$

The growth time required for the fatal disturbance (from reaching $R_{\text {crit }}$ until breakup) can be approximated by:

$$
t_{g} \approx 1.4 / \dot{\varepsilon}
$$

The exact (graphical) representation of $R_{\text {drops }}$ and $t_{g}$ can be found in Janssen et al (23).

In practice, drop sizes are generally larger than predicted by this theory. Apart from coalescence of the drops formed, one of the possible reasons is the influence of neighboring drops (or threads), of which the collision in Fig. 6 is an extreme example. Locally, a large disturbance amplitude is imposed that leads to early breakup of the thread into two parts. Subsequently, end-pinching produces drops that are larger than in the case the thread would have thinned uniformly down to $R_{\text {crit }}$. As already mentioned, this effect could be modeled in terms of the initial disturbance $\alpha_{0}$.

In contrast to viscous systems, the disintegration of viscoelastic threads has been modeled in less detail. Experiments were performed using model liquids in the opposed jets device $(24,25)$. Instead of sinusoidal, the disturbances are dumbbell shaped: spheres connected by filaments. The drainage flow from the filaments into the spheres almost stagnates because of the buildup of orientational stresses. As a consequence, thread breakup is retarded, as compared with viscous systems. In polymer blending, the

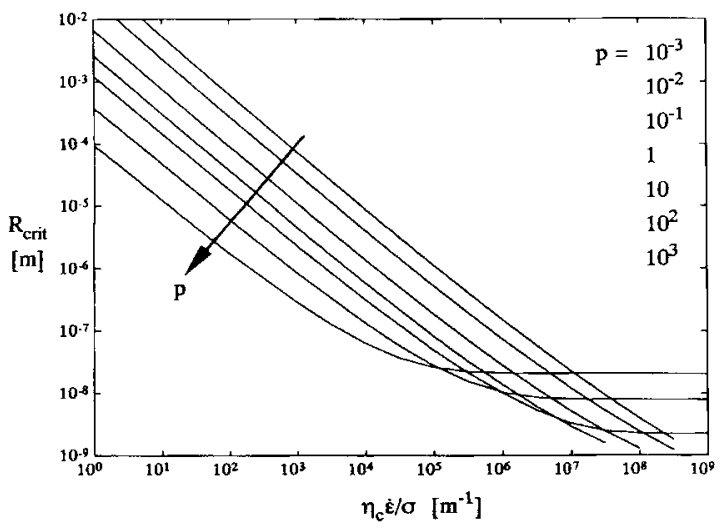

Fig. 5. Critical thread radius indicating the start of growth of the disturbance that leads to breakup of a stretching thread as a function of the modified stretching rate; parameter is the viscosity ratio $p ; \alpha_{0}=10^{-9} \mathrm{~m}$.

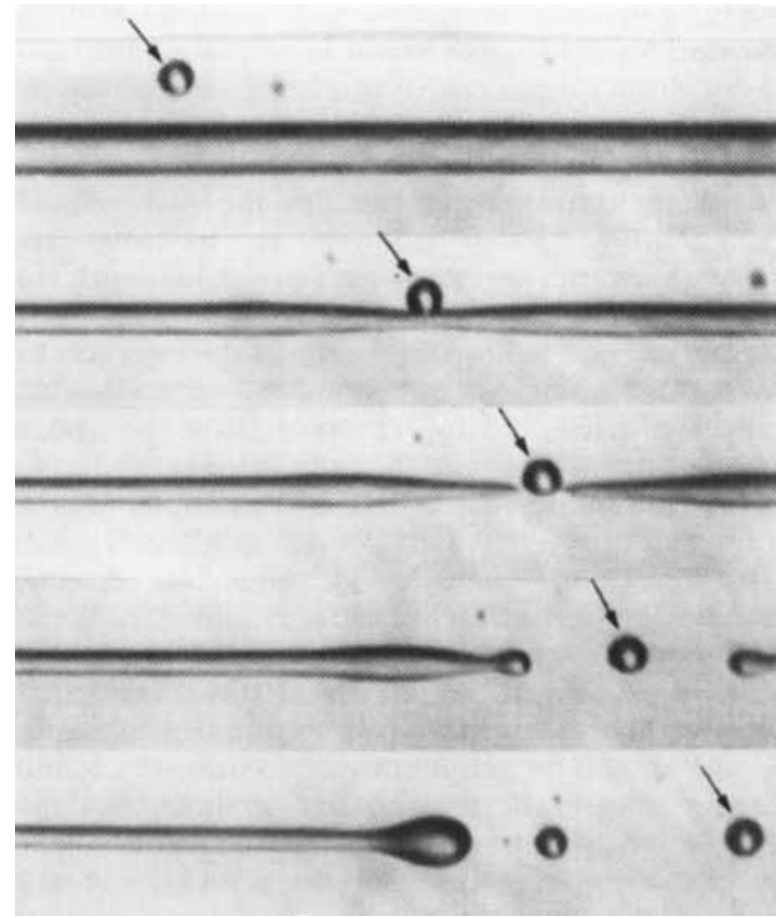

Fig. 6. Collision of a drop ( $0.2 \mathrm{~mm}$ diameter) with an extend ing thread; early "scission" leads to end-pinching, producing relatively large drops. Both liquids are viscous with $p=0.35$; photographs after every 2 seconds.

retardation of thread breakup might be generally less dramatic than in these experiments where polymer solutions were used since for a significant contribution from viscoelasticity, two criteria have to be fulfilled: i) the deformation must be applied fast compared with the relaxation time $\vartheta$ of the fluid $(\varepsilon \vartheta>1)$, and ii) the total strain must be sufficient ( $\dot{\varepsilon} t>1)$.

\section{Drop Breakup}

After stretching and disintegration of threads into drops, these finally (at $R \sim \mu \mathrm{m}$ ) may be subject to further deformation and breakup depending on the local capillary number and the time available. Excellent reviews on drop deformation and breakup exist in the literature (26-28). Grace's experimental study (2) was mentioned in the Introduction in order to introduce the parameter $\mathrm{Ca}_{\text {crit }}$ (Fig. 1). The drop size resulting from drop breakup at $\mathrm{Ca}_{\mathrm{crit}}$ into two equal parts reads:

$$
R_{\mathrm{drops}}=\frac{C a_{\mathrm{crit}}}{\eta_{c} \dot{\gamma} / \sigma} 2^{-1 / 3}
$$

but is not very relevant since the breakup time required may be quite long (in principle: $t_{b}=\infty$ exactly at $\mathrm{Ca}$ crit .

Independent of the flow type, the minimum in $\mathrm{Ca}$ crit is found around $p \approx 1$. As emphasized by Janssen et al (23), this does not necessarily imply that always at $p \approx 1$ the finest morphology is obtained, since a dispersion mechanism via stepwise equilibrium breakup 
at $\mathrm{Ca}_{\text {cril }}$ (the typical experiment underlying Fig. 1) is unlikely to occur in a practical situation. A transient dispersion mechanism of thread breakup during stretching generally gives smaller drops: owing to the stabilizing effect of stretching, the thread may become extremely thin before breakup occurs. The latter mechanism predicts size reduction upon increasing the viscosity ratio even above unity (see Fig. 5).

With respect to the effect of viscoelasticity on drop deformation and breakup, contradictory results are reported in the literature. Generally, it is assumed that viscoelasticity of both the drop and the matrix phase retards deformation and breakup. However, the change in $C a_{\text {crit }}$ is not too significant (29-31). In quasi-equilibrium drop deformation experiments, the deformation rate is probably too small to ensure $\varepsilon \vartheta>$ 1 so that viscoelasticity is not as significant as in thread breakup where orientational stresses are generated during the formation of the thread.

\section{COALESCENCE}

\section{Collision of Drops}

Thus far, the dispersion of isolated drops and threads has been discussed. Most practical applications, however, involve blends that are sufficiently concentrated to exhibit coalescence of the drops formed. In the modeling of coalescence, two aspects must be considered: i) do drops collide within a given process time? and ii) will a collision be successful, i.e., does the film between the drops drain sufficiently during the interaction time available to get film rupture and coalescence? The "external flow" and the volume fraction dispersed phase govern the collision frequency. In addition, the external flow provides the boundary conditions for the film drainage or "internal flow," viz., the contact force and the interaction time.

For equal spheres following the basic external flow, the collision frequency can be estimated $(32,33)$. In simple shear flow the collision frequency of an individual drop reads:

$$
C=\frac{32}{3} \dot{\gamma} R^{3} n
$$

where $n$ is the number of drops per volume that can be expressed in terms of the volume fraction dispersed phase $\phi$ :

$$
\phi=n \frac{4}{3} \pi R^{3}, \text { hence } n=\frac{3 \phi}{4 \pi R^{3}}
$$

Elimination of $n$ in $E q 11$ yields the collision frequency of a drop as a function of the shear rate and the volume fraction:

$$
C=\frac{8}{\pi} \dot{\gamma} \phi
$$

independent of the drop radius. So, on average a drop collides after every time:

$$
t_{\text {coll }}=\frac{1}{C}=\frac{\pi}{8 \dot{\gamma} \phi}
$$

In other words, a drop collides after every deformation $\gamma=\dot{\gamma} t_{\text {coll }}=\pi / 8 \phi$.

The number of collisions that an individual drop experiences during the process time $t_{\text {proc }}$ available is simply given by the ratio $t_{\text {proc }} / t_{\text {coll }}$. Evidently, the number of collisions increases with the shear rate applied and the volume fraction, whereas it is independent of the drop size. Note that the expression for $t_{\text {coll }}$ is idealized since in reality, complications arise, such as varying collision angle, nonspherical drop shape, and polydispersity (in extreme, Fig. 6).

\section{Film Drainage}

The second part of the modeling of coalescence deals with drainage of the liquid film between the colliding drops. Initially, at large separation the drops approach according to the velocity gradient of the external flow field. At a certain separation $\left(h_{0}\right)$ hydrodynamic interaction becomes significant (the drops "notice" each other) and the collision starts. The driving force for film drainage is now the contact force $F$ that acts during the interaction time $t_{\text {int }}$. The drops become flattened and the drainage rate decreases: the film thickness $h$ asymptotically decays to zero. However, when a critical film thickness $\left(h_{\text {crit }}\right)$ is reached, instabilities grow at the interfaces, and suddenly film rupture occurs: the drops coalesce. If the interaction time of the collision is insufficient to reach $h_{\text {crit }}$, the drops do not coalesce but tumble and separate.

Several theories are available to model the process of film drainage. They differ in terms of the mobility of the interfaces: immobile, partially mobile, or fully mobile interfaces (33). Assuming i) deformable drops with a parallel-sided film, ii) a constant contact force $F$, and iii) viscous flow, the required drainage time (from $h_{0}$ down to $h_{\text {crit }}$ ) for immobile interfaces reads:

$$
t_{\mathrm{drain}} \sim \frac{3 \eta_{\mathrm{c}} R^{2} F}{16 \pi \sigma^{2}}\left(\frac{1}{h_{\mathrm{crit}}^{2}}-\frac{1}{h_{\mathrm{o}}^{2}}\right)
$$

for partially mobile interfaces:

$$
t_{\text {drain }} \sim \frac{\pi \eta_{d} F^{1 / 2}}{2(2 \pi \sigma / R)^{3 / 2}}\left(\frac{1}{h_{\text {cril }}}-\frac{1}{h_{0}}\right)
$$

and for fully mobile interfaces:

$$
t_{\text {drain }} \sim \frac{3 \eta_{c} R}{2 \sigma} \ln \left(\frac{h_{0}}{h_{\text {crit }}}\right)
$$

The contact force $F$ is the Stokes drag force acting on the drops; its order of magnitude can be estimated from the external simple shear flow considered:

$$
F \sim 6 \pi \eta_{c} \dot{\gamma} R^{2}
$$

although in reality it is not constant during the collision. The initial film thickness $h_{0}$ corresponds to the separation at which the constant approach velocity at 
large separations $(\dot{\gamma} R)$ equals the decaying approach velocity from the drainage models. For immobile interfaces $h_{0}$ can thus be approximated by (6):

$$
h_{0} \sim R\left(\frac{3}{2} C a\right)^{2 / 3}
$$

for partially mobile interfaces:

$$
h_{0} \sim R\left(\frac{3}{16}\right)^{1 / 4} p^{1 / 2} \mathrm{Ca}^{3 / 4}
$$

and for fully mobile interfaces:

$$
h_{\mathrm{o}} \sim R \frac{3}{2} \mathrm{Ca}
$$

The critical film thickness $h_{\text {crit }}$ at which sudden rupture of the film and thus coalescence occur is governed by van der Waals forces destabilizing the thin film; it can be approximated by (33):

$$
h_{\text {crit }} \sim\left(\frac{A R}{8 \pi \sigma}\right)^{1 / 3}
$$

with $A$ the material-dependent Hamaker constant (typically $10^{-20} \mathrm{~J}$ ). For $\mu \mathrm{m}$-sized drops with $\sigma=5 \times$ $10^{-3} \mathrm{~N} / \mathrm{m}$, the value of $h_{\text {crit }}$ is about $5 \times 10^{-9} \mathrm{~m}$. Its dependence on the drop radius is fairly weak.

From $E q 15$ it is clear that all three models predict that larger drops need more time to drain the film. This is mainly due to the smaller pressure peak at the center of the film at larger drop radii; in addition, larger drops give a larger flattened area so that there is more liquid to drain. The consequence is that preferentially small drops coalesce: above a certain drop size they do not coalesce under given conditions although collisions may take place. Interfacial tension makes the drops more spherical, resulting in a smaller flattened area and a shorter drainage time. The model with partially mobile interfaces is the only one that includes the viscosity of the dispersed phase (and thus the viscosity ratio $p$ ).

Furthermore, it is interesting to note that according to two models, a larger value of $F$ leads to slower drainage of the film. The reason is the larger flattened area between the drops upon a larger contact force (viz., with $a$ the radius of the flattened area, it follows from the pressure balance $F / \pi a^{2} \sim 2 \sigma / R$ that $a \sim$ $\checkmark(F R / 2 \pi \sigma)$ or, using $\left.E q 16, a \sim R_{\sqrt{ }}(3 C a)\right)$. Hence, at larger $F$ (or $C a$ ), more liquid has to drain over a larger distance, which, of course, increases the drainage time. An important consequence is that coalescence preferentially takes place in relatively quiet zones of the mixer with soft collisions $(\rightarrow$ small drainage time required and large interaction time available) and not in regions with high deformation rate $(\rightarrow$ large drainage time required and small interaction time available). The interaction time, i.e., the duration of the collision, can be approximated from the external shear rate:

$$
t_{\mathrm{int}} \sim 1 / \dot{\gamma}
$$

Thus far, collisions of equally sized drops have been considered. For unequal drops, the same equations for the film drainage hold, provided that an equivalent drop radius is used (34):

$$
R_{\mathrm{eq}}^{-1}=\left(R_{1}^{-1}+R_{2}^{-1}\right) / 2
$$

so that $R_{\mathrm{eq}}$ ranges from $R_{1}$ (if $R_{2}=R_{1}$ ) to $2 R_{1}$ (if $R_{2}=\infty$ ). The transitions between the different drainage models follow from the restrictions of the partially mobile interfaces model (35). In essence, for small $p\left(<6 h_{\text {crit }} / a\right)$, the drops can be considered inviscid, yielding fully mobile interfaces, while for large $p\left(>3 a / h_{\text {crit }}\right)$, the drops become rigid with immobile interfaces.

A few simplifications can now be introduced. First, the terms $1 / h_{0}^{2}$ and $1 / h_{0}$ in $E q 15 a, b$ are neglected, since generally $h_{\mathrm{o}} \gg h_{\text {crit }}$; in Eq $15 c, h_{0}$ is approximated by the drop radius $R$. Second, the value of $h_{\text {crit }}$ is taken constant at $5 \times 10^{-9} \mathrm{~m}$.

\section{The Coalescence Window}

To decide whether coalescence will occur in a given situation, the relevant time scales must be compared: if $t_{\text {proc }} \geq t_{\text {coll }}$, a collision takes place that leads to coalescence only if $t_{\text {int }} \geq t_{\text {drain }}$ at the same time. This can be expressed in the following definition of the coalescence probability $P_{\text {coa }}$ being the product of the collision and the drainage probability:

$$
P_{\text {coa }}=P_{\text {coll }} P_{\text {drain }}=\exp \left(-\frac{t_{\text {coll }}}{t_{\text {proc }}}\right) \exp \left(-\frac{t_{\text {drain }}}{t_{\text {int }}}\right)
$$

Each of the constituting probabilities is of the form $P=\exp \left(-t_{\text {required }} / t_{\text {available }}\right)$ and ranges from 0 (if $t_{\text {required }}>t_{\text {available }}$ ) to 1 (otherwise). Using the expressions for the different time scales as presented, the coalescence probability for the drainage model with partially mobile interfaces reads:

$$
P_{\text {coa }}=\exp \left(-\frac{\pi}{8 \dot{\gamma} \phi t_{\text {proc }}}-\frac{\sqrt{3}}{4} \frac{R}{h_{\text {crit }}} p C a^{3 / 2}\right)
$$

and is presented graphically in Fig. 7. The "coalescence window" is evident. For too small capillary numbers (or shear rates), no collisions take place within the process time available $\left(t_{\text {proc }}\right)$. At larger $\mathrm{Ca}$ values, collisions do take place and are "soft" enough to get the film drained within the duration of the collision $\left(t_{\text {int }}\right)$, thus leading to coalescence. Finally, if $\mathrm{Ca}$ is too large, numerous collisions take place, but these are too fast to get the film drained so that coalescence will not occur. Consequently, only for a bounded interval of $\mathrm{Ca}$, coalescence is to be expected. The width of this coalescence window decreases for larger drops and even disappears for drops that are too big. Moreover, it can be seen that coalescence takes place at values of the capillary number ( $\mathrm{Ca} \sim$ $10^{-3}$ ) that are far too small for drop breakup (compare with Fig. 1, where $C a_{\text {crit }} \sim 1$ or larger). Consequently, coalescence takes place in quiet regions of a 


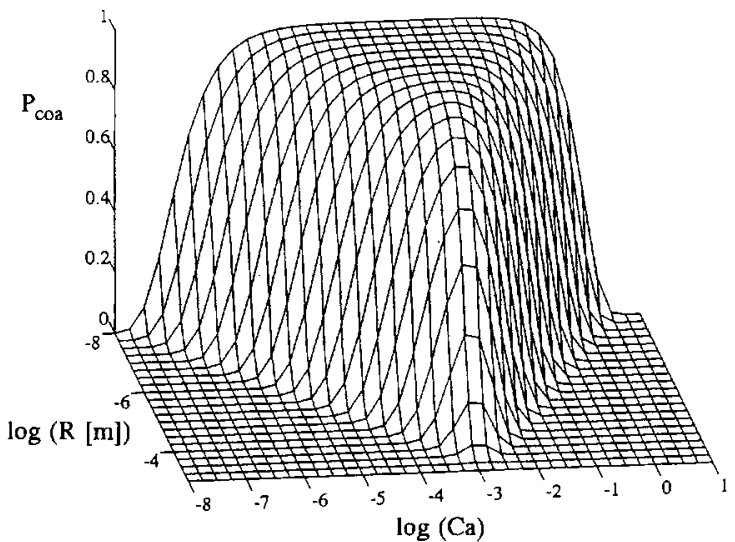

Fig. 7. Coalescence probability as a function of capillary number and drop radius. Conditions: simple shear flow, $t_{\text {proc }}=50 \mathrm{~s}, \eta_{d}=\eta_{\mathrm{c}}=1 \mathrm{~Pa} \quad \mathrm{~s}, \sigma=10^{-2} \mathrm{~N} / \mathrm{m}, h_{\text {crit }}=5 \times$ $10^{-9} \mathrm{~m} . \phi=0.1$, drainage with partially mobile interfaces.

mixer, whereas stretching of drops occurs in regions with high deformation rates. For different viscosities, the peak in Fig. 7 shifts only slightly, so that roughly the same picture holds.

\section{Comparison of Breakup and Coalescence}

In order to compare the distinct criteria for drop breakup and coalescence, a simple shear flow is considered that acts sufficiently long to reach the final equilibrium state (i.e., $t_{\text {proc }}>t_{\text {coll }}$ is ensured). The occurrence of coalescence is now simply given by the equality $t_{\text {int }}=t_{\text {drain }}$. Elaboration yields a critical drop size below which coalescence takes place and above which it does not. For the drainage model with immobile interfaces:

$$
R=\left(\frac{8}{9}\right)^{1 / 4} h_{\text {crit }}^{1 / 2}\left(\frac{\eta_{c} \dot{\gamma}}{\sigma}\right)^{-1 / 2}
$$

for partially mobile interfaces:

$$
R=\left(\frac{4}{\sqrt{3}} h_{\text {crit }}\right)^{2 / 5} p^{-2 / 5}\left(\frac{\eta_{c} \dot{\gamma}}{\sigma}\right)^{-3 / 5}
$$

and for fully mobile interfaces $R$ has to be solved from:

$$
R \ln \left(\frac{R}{h_{\text {crit }}}\right)=\frac{2}{3}\left(\frac{\eta_{c} \dot{\gamma}}{\sigma}\right)^{-1}
$$

Evidently, the radius of the drops formed $\left(R_{\mathrm{drops}}\right)$ is $2^{1 / 3}(=1.26)$ times that of the original drops $(R)$. The breakup criterion for stepwise equilibrium drop breakup is obtained from the critical capillary number ( $E q 10)$.

In Fig. 8 the maximum drop size resulting from coalescence is given for the three different drainage models $(p=1)$. It is clear that a higher mobility of the interfaces favors film drainage and thus coalescence. The (steepest) full line gives the smallest possible drop size from stepwise equilibrium breakup in simple shear. It is clear that drops that are too large break while those that are too small coalesce. The

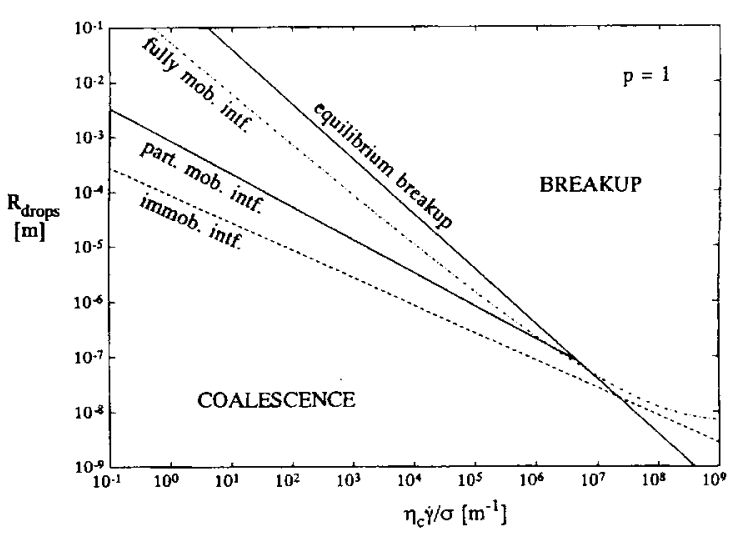

Fig. 8. Comparison of drop sizes resulting from stepwise equilibrium breakup and coalescence after long process time in simple shear.

area between the breakup curve and (one of) the coalescence curves gives the possible range of drop sizes after long process time as a function of the flow parameter $\eta_{c} \dot{\gamma} / \sigma$. In most cases the model with partially mobile interfaces is the most realistic for polymer melt blending. For viscosity ratios $p$ deviating from unity, the picture looks quite similar: only the full lines ( $E q 1 O$ and $23 b$ ) depend on $p$. Generally, the "steady state" drop size decreases almost proportionally with the flow parameter. Especially the narrowing of the drop size range at larger shear rates could be checked experimentally, e.g., using a Couette device.

Surfactants (or compatibilizers) present in or on the drop phase may immobilize the interface and thus retard film drainage and prevent coalescence. Surfactants present in the continuous phase are generally much less effective since they cannot reach the interface as easily (36). A complete analysis of the film drainage with a surfactant includes the convection/diffusion problem giving concentration and thus interfacial tension gradients. In specific cases, surfactants may also suppress coalescence by steric hindrance. These aspects are subjects of the current research in our laboratory.

Apart from the prevention of coalescence, the finer morphology usually experienced upon addition of surfactants is due to a decrease of the interfacial tension $\sigma$, inducing i) a delay of thread breakup allowing for thinner threads prior to breakup and ii) an increase of the local capillary number allowing for breakup of smaller drops.

Viscoelasticity of the continuous phase is expected to retard film drainage since (biaxial) elongational viscosities may build up in the squeezing film. Viscoelasticity of the dispersed phase will have a much smaller effect.

\section{A 2-ZONE MIXING MODEL}

\section{Principle}

In the preceding sections, the most relevant stages of the compounding process of immiscible liquids 
have been discussed. The resulting criteria for Newtonian liquids are quite complete and can be combined into an overall mixing model that describes the development of a morphology during compounding. A schematic 2-zone model has been chosen that has an analogy with the mixing model for carbon black in rubber as proposed more than a decade ago by Manas-Zloczower et al. (37). The 2-zone model is depicted in Fig. 9. The material passes cycles through alternating "strong" and "weak" zones. A diversity of practical mixing devices can be modeled by variation of the flow conditions in these zones (residence times and deformation rates) and by the total number of cycles.

The strong zone represents regions of a mixer where high deformation rates are present. It is modeled by elongational flow with high elongation rate $\dot{\varepsilon}$ and short residence time. In this zone, exponential stretching of dispersed drops and threads takes place unless drops with $\mathrm{Ca} / \mathrm{Ca}_{\text {crit }}<5$ are considered. During stretching it is checked (using Fig. 5) if the threads formed are thin enough to disintegrate in the flow. Although the strong zone is modeled by elongational flow, simulating, e.g., the entrance of a narrow gap before a kneading disc, it may also represent a sequence of stretching and folding operations in an effective simple shear region (Fig. 3) since in both cases stretching proceeds exponentially.

The weak zone represents quiescent regions of the mixer and is modeled by simple shear; the shear rate $\dot{\gamma}$ of the external flow is small, the residence time large. Threads entering this zone may break up at (almost) rest if the residence time suffices. Drops coalesce if the remaining residence time allows for collisions with successful film drainage. Repeated coalescence may take place during each passage of the weak zone if there is time available. In order to incorporate the typical randomness of the material flow in this region, the residence time in the weak zone is considered to be distributed as a cascade of "ideal mixers"; this is a usual residence time distribution in chemical reaction engineering (38).

In the analyses of stretching, breakup, and coalescence, the volume fraction of the dispersed phase $(\phi)$ is taken into account only in the collision frequency involved in coalescence. To incorporate the volume fraction in the total model, individual drops and threads are assumed to experience an effective ma-

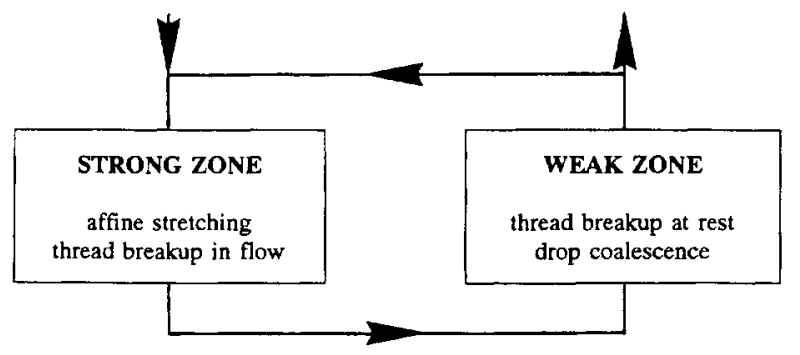

Fig. 9. Principle of the 2-zone mixing model: material passes cycles through alternating strong and weak flow zones. trix viscosity ( $\left.\eta_{c, \text { eff }}\right)$ that differs from that of the pure continuous phase component $\left(\eta_{c}\right)$ because of the presence of neighboring drops and threads. Throughout the model $\eta_{c}$ is replaced by $\eta_{c \text {, eff }}$; only in the local process of film drainage prior to coalescence is $\eta_{C}$ of the pure component maintained. As effective viscosity of the emulsion the Krieger and Dougherty equation (39) for monodisperse rigid spheres has been modified to account for fluid spheres (6):

$$
\eta_{c, \mathrm{eff}} / \eta_{c}=\left(1-\phi / \phi_{m}\right)^{-2.5 \phi_{m}(p+0.4) /(p+1)}
$$

with $\phi_{m}$ the maximum packing density. For fluid drops, $\phi_{m}$ depends on the viscosity ratio $p$ and the interfacial stress $\sigma / R$ (since both influence the deformability of the dispersed particles). Here, $\phi_{m}$ was set to 0.8 . Following $E q 24$, the effective matrix viscosity ranges from $\eta_{c}$ if $\phi=0$ to infinity if $\phi \rightarrow \phi_{m}$. From equaling the effective viscosity of the $A$ in $B$ and the $B$ in $A$ emulsions, the critical volume fraction at phase inversion can be estimated in dependence on the viscosity ratio $\eta_{B} / \eta_{A}$ of the constituents (6. 40).

\section{Numerical Approach}

Since the number of drops increases enormously ( 1 single mm-sized drop yields $10^{9} \mu \mathrm{m}$-sized drops), drop "families" are considered rather than individual drops. Each family contains a number of identical drops or threads characterized by a single radius $R$, an aspect ratio $L / B$ (with $B=2 R$ ), and a cumulative residence time. Within a family no time distribution is applied so that a number of drop families has to be used to allow for a distribution of drop sizes. It was decided to artificially initiate this distribution the exact initial morphology does not affect the steady state). Further distribution is achieved as a result of the residence time distribution in the weak zone. All drop families start in the strong zone, pass through a specified number of cycles, and finally leave from the weak zone. As each drop family passes a zone, it is checked as to whether or not the current size and flow conditions give rise to stretching, breakup, or coalescence.

Concerning the distinct stages of the mixing process, a number of assumptions have been made. Drops with $1<\mathrm{Ca} / \mathrm{Ca} a_{\text {crit }}<5$ are not deformed in the strong zone although they are unstable. The reason is that they would stretch only slowly (less than affine) while the residence time in the strong zone is short. Furthermore, affine deformation is calculated according to 3-D elongational flow, whereas values of $\mathrm{Ca}$ crit are taken from 2-D elongation. Coalescence takes place only within each drop family because it is computationally intractable to give an individual drop the opportunity to collide with all individual drops in the system; moreover, collision frequencies of unequal drops are still quite poorly modeled. By doing so, the number of collisions would be underestimated severely, which is prevented here by taking the total volume fraction $\phi$ (summarized over all drop families) 


\section{Dynamics of Liquid-Liquid Mixing: A 2-Zone Model}

to be present in every single drop family. Film drainage prior to coalescence is modeled with partially mobile interfaces unless (the before-mentioned) restrictions are met, indicating transition to immobile or fully mobile interfaces.

\section{Morphology Development}

As a typical illustration of the 2-zone model, the melt blending of polymers in an extruder is simulated. The relevant material parameters are chosen as follows: $\eta_{d}=\eta_{c}=10^{2} \mathrm{~Pa} \cdot \mathrm{s}, \sigma=5 \times 10^{-3} \mathrm{~N} / \mathrm{m}, \alpha_{0}=$ $10^{-9} \mathrm{~m}$, and $\phi=0.2$. The strong zone is modeled by $\dot{\varepsilon}=30 \mathrm{~s}^{-1}$ and $t=0.1 \mathrm{~s}$ (yielding $L / B=e^{3} / e^{3 / 2}=90$ upon affine stretching of a sphere); in the weak zone $\dot{\gamma}=3 \mathrm{~s}^{-1}$ and the residence time distribution resembles a cascade of two ideal mixers with a total mean residence time $\bar{\tau}=10 \mathrm{~s}$. Initially, a population of 100 drop families is generated with diameters around $1 \mathrm{~mm}$. Figure 10 shows the evolution of the drop size distribution as the population passes cycles through alternating strong and weak zones. The probability density is calculated after rediscretizing all 100 drop families into a smaller number of intervals; it is defined as $\left(V_{i} / V_{10 \text { tal }}\right) / \Delta(\log R)$, with $V_{i}$ the volume occupied by drop families with radius in the interval $\Delta(\log R)$ around $R_{i}$. (This rediscretization is necessary to avoid "needle-like" distributions.)

During the first three cycles of alternating strong and weak zones, all drops are stretched affinely in the strong zone: threads about $10 \mu \mathrm{m}$ thick are formed. This is too thick to break up either during stretching or at rest in the weak zone (given the limited residence time); so, nothing happens in the weak zone. The extremely large aspect ratios of course must represent packages of folded threads (see Fig. 3). During the fourth cycle, after further stretching in the strong zone, some of the threads break up into droplets $(L / B=1)$ in the weak zone. The breakup time required at rest has decreased with the radius ( $E q$ 6) and has now reached the mean residence time in the weak zone. In the fifth cycle, the drops and remaining threads are stretched again. All threads formed are thin enough to disintegrate during the residence time available in the weak zone. At this point, submicron drops appear that may easily coalesce: the lower limit of the drop size is reached. After six cycles a dynamic steady state is obtained: drops that are too small coalesce (repeatedly); drops that are too large stretch and break again.

The simulation is supported by existing experimental studies. For example, in the work of Elemans (41), the blending of PS/HDPE on a co-rotating twin-screw extruder (25-mm screw diameter) yields a typical length scale in the $\mu \mathrm{m}$ range after a similar residence time in the extruder. Evidently, the effect of neglecting viscoelasticity in the present 2 -zone mixing model is not too severe for a rough simulation of the blending of polymer melts, as already expected from the treatment of the separate processes that occur. As mentioned, the strong zone represents both a convergence before passing a kneading flight and linear stretching in simple shear dominated flow regions accompanied by folding. Generally, (twin-screw) extruders contain a large number of kneading discs, but, depending on the gap height between flight tips, and barrel wall, only a fraction of the material indeed passes through the narrow gap. The weak zone represents transport through the screw channel, typically at low deformation rate.

\section{Influence of Material Parameters}

As a guideline in investigating the influence of different viscosities, first the scaling laws for the distinct stages are given (valid under limited conditions). For drop stretching and breakup, governed by $\mathrm{Ca} / \mathrm{Ca}_{\text {crit }}, \mathrm{Eq} \mathrm{I}$ and Fig. 1 show that for $p \approx 1: R \propto$ $\eta_{c}^{-1} p^{0}$ or $\eta_{c}^{-1}$. For thread breakup; during stretching, Fig. 5 and $E q 8$ indicate that: $R \propto \eta_{c}^{-0.9} p^{-0.45}$ or $\left(\eta_{d} \eta_{c}\right)^{-0.45}$. For coalescence with partially mobile interfaces $E q 23 b$ and 18 yield: $R \propto \eta_{c}^{-9 / 13} p^{-6 / 13}$ or $\left(\eta_{d}^{2} \eta_{c}\right)^{-0.23}$. For thread breakup at rest, Eq 7 and Fig. 4 (around $p \approx 1$ ) show that the resulting drop radius depends mainly on the initial radius and only slightly on the $p$-dependent wave number; hence, scaling between $R$ and $\eta_{d . c}$ is only of little use here. However, concerning the required breakup time of a thread at rest, Eq 6 and Fig. 4 (for $0.1<p<4$ where $\Omega_{m} \propto p^{-0.5}$ ) lead to: $t_{b} \propto \eta_{c} p^{0.5}$ or $\left(\eta_{d} \eta_{c}\right)^{0.5}$.

From these proportionalities it is evident that higher viscosities (either $\eta_{d}$ or $\eta_{c}$ ) favor a thread-like dispersion route (larger time required for thread breakup) with suppression of coalescence; hence, finer morphologies are obtained. For example, in Fig. 8 the decrease of the drop size with increasing $\eta_{c}$ is evident. However, the smaller the drops, the more dominant coalescence becomes, giving a lower boundary for the drop size (about $10^{-7} \mathrm{~m}$ for the processing conditions of Fig. 10).

For a fixed value of $\eta_{c}$, it is interesting to investigate the influence of $\eta_{d}$ (and thus $p$ ). Figure 11 shows the steady state drop sizes (after ten cycles) for three different $\eta_{d}$ values and the residence time distribution that is characteristic for all three cases. [The probability density for $t$ is defined as: $\left.\left(V_{i} / V_{\text {total }}\right) / \Delta t\right]$. The drop size distribution for $p=1$ corresponds to Fig. 10 and confirms that the result after six cycles is indeed the (dynamic) steady state. Keeping $\eta_{c}$ constant, a larger $\eta_{d}(p=100)$ promotes finer dispersion, owing to retardation of both thread breakup (allowing for further stretching) and coalescence (interfaces actually become immobile). The dispersion route is mainly the one-step breakup of highly extended threads. Upon a decrease of $\eta_{d}(p=0.01)$, threads break up more quickly, implying a stepwise stretching and breakup route. In addition, the smaller value of $\eta_{d}$ favors coalescence, resulting in coarser morphologies. It should be noted that varying $p$ by means of $\eta_{c}$ (at constant $\eta_{d}$ ) gives a result different from Fig. 11: decreasing $\eta_{C}$ simply yields coarser morphologies (although p rises); compare Figs. 5 and 8 . So, both the absolute level and the ratio of the viscosities matter. Similar effects of the viscosity 
Jos M. H. Janssen and Han E. H. Meijer
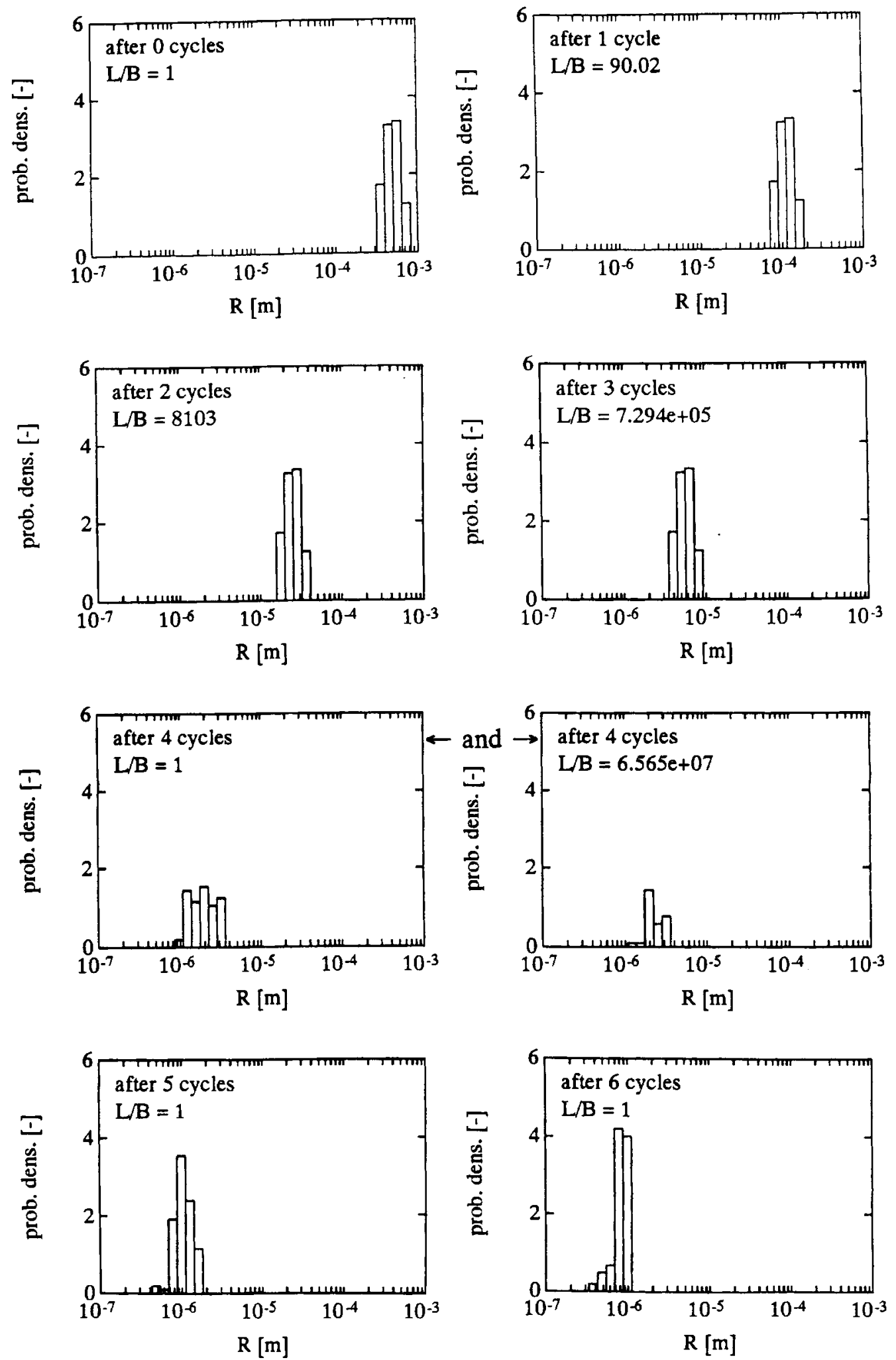

Fig. 10. Morphology development in the 2-zone mixing model with: $\eta_{d}=\eta_{\mathrm{c}}=10^{2} \mathrm{~Pa} \quad \mathrm{~s}, \sigma=5 \times 10^{-3} \mathrm{~N} / \mathrm{m}, \alpha_{0}=10^{-9} \mathrm{~m}$, and $\phi=0.2$; strong zone: $\dot{\varepsilon}=30 \mathrm{~s}^{-1}$ and $t=0.1 \mathrm{~s}$; weak zone: $\dot{\gamma}=3 \mathrm{~s}^{-1}$ and $t=10 \mathrm{~s}$. Note that after four cycles, two distributions with different $L / B$ apply. 
Dynamics of Liquid-Liquid Mixing: A 2-Zone Model
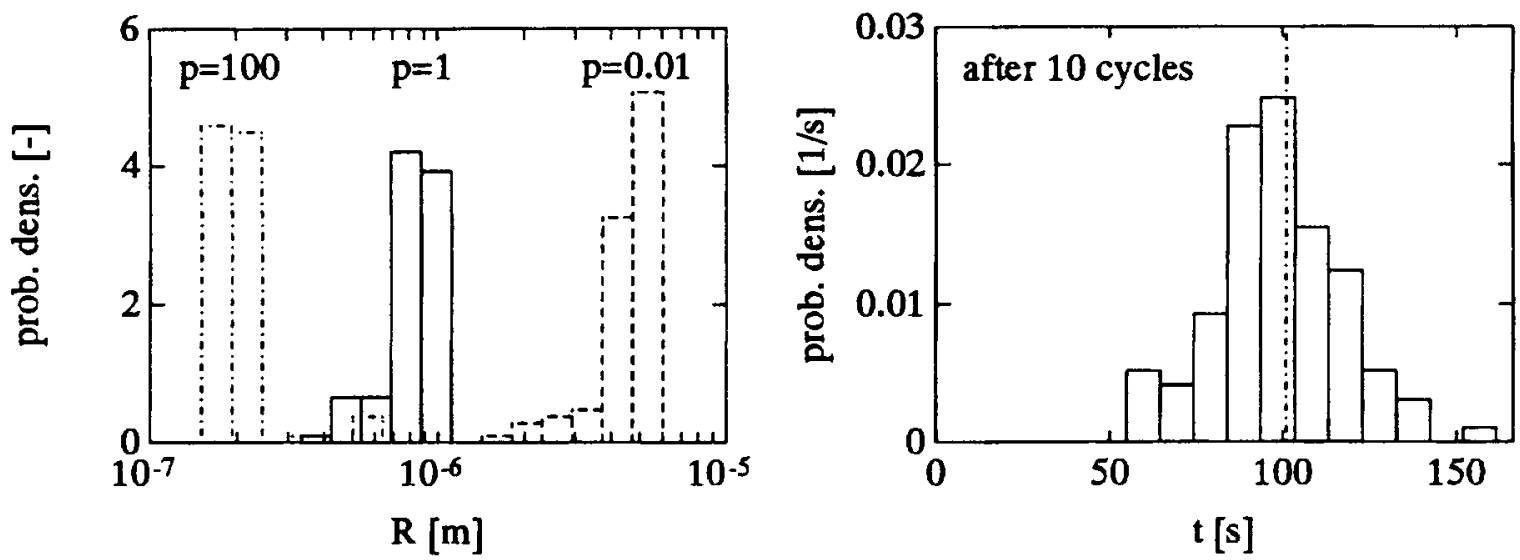

Fig. 11. Left: Steady-state morphologies from the 2-zone model with: $\eta_{c}=10^{2} \mathrm{~Pa} \cdot \mathrm{s}$ and $\eta_{d}=10^{4}, 10^{2}$, or $1 \mathrm{~Pa} \cdot \mathrm{s}\left(\mathrm{p}=\eta_{d} / \eta_{c}=10^{2}\right.$, $\left.1,10^{-2}\right)$; other conditions are the same as in Fig. 10. Right: Residence time distribution characteristic for all three cases.

(ratio) on the dispersion route and the drop size were reported by Ottino and co-workers $(42,43)$.

The commercial blend Noryl GTX (General Electric Plastics) is a practical example where a large viscosity ratio $p$ indeed leads to a fine dispersion. Essentially, a highly viscous polyphenylene ether melt is dispersed in a much lower viscous polyamide continuous phase $\left(p=\eta_{d} / \eta_{c} \approx 20\right)$, but still the total system is rather viscous $\left(\eta_{c} \sim 200 \mathrm{~Pa} \cdot \mathrm{s}\right)$. Just looking at the critical capillary number for drop breakup (Fig. 1) suggests that a fine dispersion cannot be obtained since the viscosity ratio $p$ is far above 1 . However, taking into account all mechanisms and time scales such as in the 2-zone model, it becomes clear that this blend can easily be dispersed to a length scale of $10^{-7} \mathrm{~m}$, which is reality. It should be noted that this commercial blend of course contains many additives, including a rubbery third phase and compatibilizers.

A decrease in interfacial tension (as caused by addition of compatibilizers) yields a proportionally finer morphology: $\sigma / R$ affects all stages in a similar way. Only coalescence is favored less than proportionally with $\sigma$ ( $E q 23 b$ and 18: $R \propto \sigma^{9 / 13}$ ) causing the lower bound of the drop size. The volume fraction $\phi$ affects the model both via the enlarged effective matrix viscosity and the collision frequency for coalescence: generally, a larger $\phi$ gives a coarser morphology. Moreover, in reality, a larger $\phi$ generates a higher level of initial disturbances $\alpha_{0}$ because of the pushing of neighboring drops and threads. This accelerates thread breakup and makes the dispersion route more stepwise, resulting in a coarser morphology. However, simulations with varying $\alpha_{0}$ show only a slight dependency, because the final drop size is mainly determined by coalescence, which does not include $\alpha_{0}$.

\section{Influence of Processing Conditions}

The 2-zone model is flexible for investigating the influence of processing conditions. Increasing the strain $(\varepsilon=\dot{\varepsilon} t)$ applied in the strong zone yields thin- ner threads within a single passage. In the extreme, thread breakup during stretching results in drop sizes according to Eq 8 and Fig. 5. In more realistic cases, however, the final drop size strongly depends on the occurrence of coalescence. In the weak zone, a longer residence time favors thread breakup at rest and thus implies a stepwise breakup route. Whether coalescence is also favored by a longer residence time in the weak zone depends on the shear rate (the "coalescence window" in Fig. 7).

An interesting example of varying the processing conditions is the optimization of the gap height $\delta$ between screw or kneading flight and barrel wall. By decreasing the gap height, the converging flow at the gap entrance becomes more effective for stretching, but at the same time less material will pass through. Two extreme cases are i) $\delta=0$ so that $\dot{\varepsilon}=\infty$ but no material can pass, and ii) $\delta=H$ (the channel depth), i.e., no flight at all, so that all material will pass, however, with $\dot{\varepsilon}=0$. Somewhere between these extremes, an optimal gap height $\delta / H$ exists. In Fig. 12, such an optimization is illustrated based on the 2 -zone mixing model. The resulting mean drop size is calculated as a function of $\delta / H$. Therefore, the processing conditions are varied as follows. As a reference case the calculation of Fig. 10 after six cycles is used with identical material parameters $\left(\eta_{d}=\eta_{c}=10^{2}\right.$ $\mathrm{Pa} \cdot \mathrm{s}, \sigma=5 \times 10^{-3} \mathrm{~N} / \mathrm{m}, \alpha_{0}=10^{-9} \mathrm{~m}$, and $\phi=0.2$ ). In the strong zone (the gap entrance the strain is determined by the compression from $H$ to $\delta: \varepsilon=$ $-2 \ln (\delta / H)$ since $\delta / H=e^{-\varepsilon / 2}$. (In the reference case $\varepsilon=3$, which corresponds to $\delta / H=0.223$.) Taking the time in the strong zone constant at $0.1 \mathrm{~s}$, the stretching rate reads: $\dot{\varepsilon}\left[\mathrm{s}^{-1}\right]=\varepsilon / 0.1=-20 \ln (\delta / H)$. In the weak zone, the shear rate is constant: $\dot{\gamma}=3 \mathrm{~s}^{-1}$ and the mean residence time $\bar{t}=t_{\text {total }} / N$ with $t_{\text {total }}$ the (mean) total residence time that is fixed at $60 \mathrm{~s}$. The number of cycles (or flight passages) $N$ within this total mixing time is taken proportional to the relative gap height: $N \propto \delta / H$ with $N=6$ if $\delta / H=0.223$ (the reference case). 


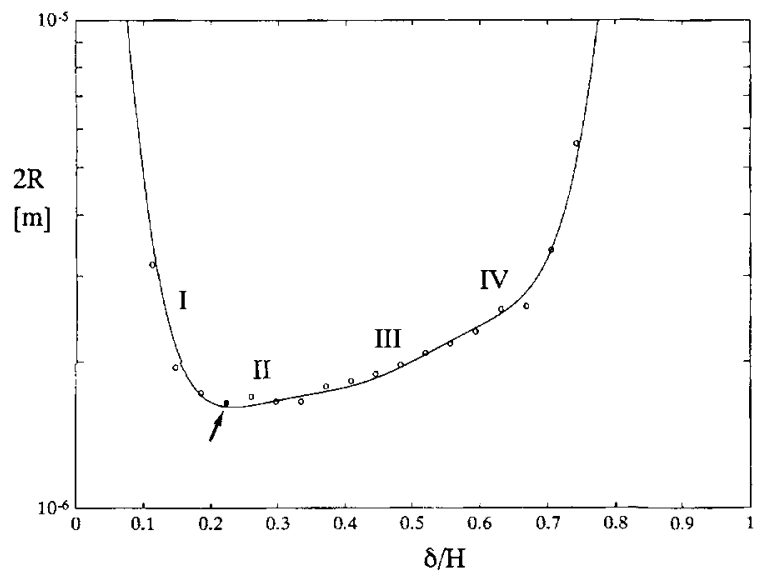

Fig. 12. Mean drop size as a function of the gap height using the 2-zone mixing model with the material parameters from Fig. 10. Symbols give results of the simulations; the curve is a fit. The arrow indicates the reference case (i.e., conditions as in Fig. 10 after six cycles).

The symbols in Fig. 12 represent the mean drop diameters resulting from the simulations; the curve is a fit. For very narrow gaps (regime I), the stretching rate is large (e.g., $\dot{\varepsilon}=44 \mathrm{~s}^{-1}$ ), but only a few cycles are performed within the residence time given (e.g., $N=3$ ). The dispersion route contains a few large stretching operations followed by final breakup. In regime II more cycles are included, while $\dot{\varepsilon}$ is still substantial (e.g., in the reference case, indicated by the arrow: $N=6$ and $\dot{\varepsilon}=30 \mathrm{~s}^{-1}$ ). The dispersion route includes stretching, breakup and coalescence. In regime III the value of $\dot{\varepsilon}$ becomes quite small but the number of cycles is large enough to obtain a steady state morphology. In regime IV, although $N$ is large (e.g., $N=20$ ), the strain per passage is so small that it takes this many cycles to thin the stretching threads down the $\mu \mathrm{m}$ range. At the same time, the residence time in the weak zone becomes so short (e.g., $t=3 \mathrm{~s})$ that only very thin threads $(R<1 \mu \mathrm{m}$ break up. If, in the simulations underlying Fig. 12, threads result after the last cycle, they are broken artificially to get drops. (Thus, mixing ends with a long stay in a weak zone in all cases.) The presence of an optimum gap height $\delta / H$ of about 0.25 is evident. For large $\delta / H$ values, however, the simulations are questionable since some basic assumptions do not hold anymore. In particular, the strong zone becomes weaker and loses its character. Irrespective of the exact values, the main message from this exercise is that "worn" extruder screws or kneader wings might be superior to new (more closely fitting ones, as pointed out by Manas-Zloczower et al. (37). Moreover, this suggests that in scaling up of a compounder, the gap should be scaled accordingly in order to keep $\delta / H$ optimal (instead of minimizing it).

Another point of consideration is the quenching history of the blend after leaving the compounder. In the examples treated, the blending process ended with a quite long stay in the weak zone, which re- sulted in a drop-in-matrix morphology. Practical polymer blending may also yield thread-in-matrix structures, resulting from quick quenching of a strand after it leaves the extruder die. In terms of the 2-zone mixing model, the die is just an extra strong zone and the stage between the die and the quenching bath another weak zone. A simple estimation of thread breakup times shows that in polymer blending, thread-in-matrix morphologies in the $\mu \mathrm{m}$ range can easily be obtained: $t_{b}$ is of the order of seconds so that quenching may take place either before or after breakup. As an additional point, the time before quenching should be minimized in order to avoid coalescence in the almost quiescent strand ( $\mathrm{Ca}$ very small, see Fig. 7). This illustrates that the quenching procedure may largely influence the final morphology as confirmed experimentally [see, e.g., Fig. 41 in Meijer and Janssen (5)].

\section{CONCLUSION}

During stretching of dispersed domains, the combined action of folding is crucial for obtaining an exponential decrease of the length scale. Breakup of viscous threads both in a quiescent matrix and during stretching can be calculated. Interestingly, for identical final deformation rates, a transient one-step process of breaking a highly extending thread yields smaller drops than the repeated breakup of drops under equilibrium conditions (at $C a_{\text {crit }}$ ) as induced by slowly increasing the deformation rate until its final (maximum) value is reached. Moreover, the transient one-step mechanism predicts a finer dispersion upon increasing either viscosity (dispersed or continuous phase). This is quite different from the commonly accepted rule of thumb that both viscosities should match (which is based on the experiments by Taylor and Grace following the stepwise equilibrium route).

For coalescence it is shown that although a larger velocity gradient implies a higher collision frequency, these fast collisions generally do not lead to coalescence since the short interaction time is insufficient for drainage of the liquid film between the colliding drops: At large velocity gradients, the drops tumble and separate. Consequently, coalescence preferentially takes place in quiescent regions of a mixing device and between small drops, while stretching occurs in strong flow regions and with large drops.

A 2-zone mixing model combines a largely schematized flow field with the relevant mixing rules and criteria. It is a useful tool for simulating the morphology development during compounding. The influence of the material parameters and processing conditions on the mixing process provides guidelines for the preparation of specific morphologies and for the optimization of compounding equipment. To attain a fine morphology, thread breakup during stretching should be aimed at. Especially high viscosities of both phases induce this mechanism and suppress coalescence. Low viscosities favor a stepwise breakup route with coalescence and lead to coarser morphologies. 


\section{NOMENCLATURE}

A

Hamaker constant $\left(\sim 10^{-20}\right)[\mathrm{J}]$.

$=$ Radius of the flattened area of a deformed drop [m].

$B \quad=$ Width of a deformed drop [m].

$C \quad=$ Collision frequency $[1 / \mathrm{s}]$.

$\mathrm{Ca}=$ Capillary number $\left(\eta_{c} \dot{\gamma} R / \sigma\right)$.

$C a_{\text {crit }}=$ Critical capillary number necessary for drop breakup.

D $=$ Rate of deformation tensor $[1 / \mathrm{s}]$.

$F \quad=$ Contact force $[\mathrm{N}]$.

$H \quad=$ Channel depth [m].

$h=$ Film thickness $[\mathrm{m}]$.

$h_{0} \quad=$ Initial film thickness [m]

$h_{\text {crit }}=$ Critical film thickness [m]

$k=$ Boltzmann's constant $\left(1.38 \times 10^{-23}\right)[\mathrm{J} / \mathrm{K}]$.

$L \quad=$ Length of a deformed drop [m].

$L_{\dot{0}} \quad=$ Initial length $[\mathrm{m}]$.

$N=$ Number of cycles.

$n \quad=$ Number of drops per volume $\left[1 / \mathrm{m}^{3}\right]$.

$P_{\text {coa }}=$ Coalescence probability.

$P_{\text {coll }}=$ Collision probability.

$P_{\text {drain }}=$ Drainage probability.

$p \quad=$ Viscosity ratio $\left(\eta_{d} / \eta_{c}\right)$.

$R \quad=$ Radius of a drop or a thread [m].

$R_{0} \quad=$ Initial radius of a drop or thread [m].

$R_{\text {crit }}=$ Critical radius at which the fatal disturbance starts growing [m].

$R_{\text {drops }}=$ Radius of drops formed [m].

$R_{\text {eq }}=$ Equivalent drop radius $[\mathrm{m}]$.

$T=$ Temperature $[\mathrm{K}]$.

$t \quad=$ Time [s].

$\bar{t}=$ Mean residence time $[\mathrm{s}]$.

$t_{b} \quad=$ Breakup time [s].

$t_{\text {coll }}=$ Required collision time [s].

$t_{\text {drain }}=$ Required drainage time [s].

$t_{g}=$ Disturbance growth time [s].

$t_{\text {int }}=$ Available interaction time [s].

$t_{\text {proc }}=$ Available process time [s].

$t_{\text {total }}=$ Total residence time [s].

$x=$ Disturbance wave number $\left(2 \pi R_{0} / \lambda\right)$.

$x_{m}=$ Dominant wave number .

$\alpha=$ Disturbance amplitude [m].

$\alpha_{0} \quad=$ Initial disturbance amplitude [m].

$\alpha_{b} \quad=$ Disturbance amplitude at breakup [m].

$\gamma \quad=$ Shear $(\dot{\gamma} t)$.

$\dot{\gamma}=$ Shear rate $[1 / \mathrm{s}]$.

$\delta \quad=$ Gap height $[\mathrm{m}]$.

$\varepsilon \quad=$ Strain $(\dot{\varepsilon} t)$.

$\dot{\varepsilon} \quad=$ Elongation or stretching rate $[1 / \mathrm{s}]$.

$\eta_{c, \text { eff }}=$ Effective viscosity of a dispersion $[\mathrm{Pa} \cdot \mathrm{s}]$.

$\eta_{d, c}=$ Viscosity of the dispersed or continuous phase $[\mathrm{Pa} \cdot \mathrm{s}]$.

$\vartheta \quad=$ Relaxation time of a fluid [s].

$\lambda=$ Disturbance wavelength [m].

$\lambda_{m}=$ Dominant disturbance wavelength [m].

$\sigma \quad=$ Interfacial tension $[\mathrm{N} / \mathrm{m}]$.

$\tau=$ Shear stress $[\mathrm{Pa}]$. $\phi \quad=$ Volume fraction dispersed phase

$\phi_{m}=$ Maximum packing density.

$\Omega_{m}=$ Dominant dimensionless growth rate.

\section{REFERENCES}

1. G. I. Taylor, Proc. R. Soc., (London) A, 146, 501 (1934).

2. H. P. Grace, 3rd Eng. Found. Conf. Mixing, Andover N.H. (1971), republished in Chem Eng. Commun., 14, 225 (1982).

3. B. J. Bentley and L. G. Leal, J. Fluid Mech, 167, 241 (1986).

4. D. V. Khakhar and J. M. Ottino, J. Fluid Mech, 166, 265 (1986).

5. H. E. H. Meijer and J. M. H. Janssen, in Mixing and Compounding - Theory and Practice, I. Manas-Zloczower and $Z$. Tadmor, eds., Progress in Pol. Processing Series, Carl Hanser Verlag, Munich (1994).

6. J. M. H. Janssen, PhD thesis, Eindhoven University of Technology, Eindhoven, The Netherlands (1993).

7. A. K. Ghosh, S. Ranganathan, J. T. Lindt, and S. Lorek, SPE ANTEC Tech Papers, 37, 232 (1991).

8. J. T. Lindt and A. K. Ghosh, Polym. Eng. Sci., 32, 1802 (1992).

9. P. H. M. Elemans, H. L. Bos, J. M. H. Janssen, and H. E. H. Meijer, Chem. Eng. Sci., 48, 267 (1993).

10. K. Y. Ng and L. Erwin, Polym. Eng. Sci., 21, 212 (1981).

11. H. Aref, Phys. Fluids A, 3, 1009 (1991).

12. C. W. Leong and J. M. Ottino, J. Fluid Mech. 209. 463 (1989).

13. J. M. Ottino, The Kinematics of Mixing: Stretching, Chaos and Transport, Cambridge Univ. Press, Cambridge, England (1989).

14. V. V. Meleshko, V. T. Grinchenko, and T. L. Isaeva, Doklady Akademii Nauk Ukrainskoj SSR ser. A, N8 (in Ukrainian), 64 (1991).

15. G. W. M. Peters and V. V. Meleshko, J. Fluid Mech, in preparation (1995).

16. P. H. M. Elemans and H. E. H. Meijer. Polym Eng. Sci.. 30, 893 (1990)

17. S. Tomotika, Proc. R. Soc., (London) A, 150, 322 (1935).

18. P. H. M. Elemans, J. M. H. Janssen, and H. E. H. Meijer. J. Rheol, 34, 1311 (1990).

19. W. Kuhn, Kollö̈d Z., 132, 84 (1953).

20. S. Tomotika. Proc. R. Soc., (London A, 153, 302 (1936).

21. T. Mikami, R. Cox, and R. G. Mason, Int. J. Multiphase Flow, 2, 113 (1975).

22. D. V. Khakhar and J. M. Ottino, Int. J. Multiphase Flow. 13, 7 I (1987).

23. J. M. H. Janssen and H. E. H. Meijer, J. Rheol. 37, 597 (1993).

24. J. M. H. Janssen, F. P. T. Baaijens, and H. E. H. Meijer. Proc. XIth Int Congr. on Rheol. 369. Brussels; Elsevier. Amsterdam (1992).

25. J. M. H. Janssen, G. W. M. Peters and H. E. H. Meijer. Chem. Eng. Sci., 48, 255 (1993).

26. A. Acrivos. 4th Int. Conf. on Physiochemical Hydrodynamics, Ann. N.Y. Acad. Sci., 404, 1 (1983).

27. J. M. Rallison, Ann Rev. Fluid Mech, 16, 45 (1984).

28. H. A. Stone, Ann Rev. Fluid Mech. 26, to appear (1994).

29. W. J. Milliken and L. G. Leal, J. Non-Newt. Fluid Mech. 40, 355 (1991).

30. W. J. Milliken and L. G. Leal, J. Non-Newt. Fluid Mech, 42, 231 (1992).

31. R. A. de Bruijn. PhD thesis, Eindhoven University of Technology, Eindhoven, The Netherlands (1989).

32. M. Smoluchowski, Z. Phys. Chem. 92, 129 (1917).

33. A. K. Chesters, Trans IChemE, 69A, 259 (1991).

34. A. K. Chesters and G. Hofman, Appl. Sci. Res., 38, 353 (1982).

35. S. Abid and A. K. Chesters, Int. J. Multiphase Flow, accepted (1993). 
36. I. B. Ivanov, Pure Appl. Chem., 52, 1241 (1980).

37. I. Manas-Zloczower, A. Nir, and Z. Tadmor, Rubber Chem. Technol, 55, 1250 (1982).

38. O. Levenspiel. Chemical Reaction Engineering. 2nd ed., Eq. 50 on p. 291, John Wiley \& Sons, New York (1972).

39. I. M. Krieger and T. J. Dougherty, Trans. Soc. Rheol, $\mathbf{3}$, 137 (1959)
40. L A Utracki, J Rheol, 35, 1615 (1991).

41. P. H. M. Elemans, PhD thesis, Eindhoven University of Techynology, Eindhoven, The Netherlands (1989).

42. M. Tjahjadi and J. M. Ottino, J. Fluid Mech, 232, 191 (1991).

43. F. J. Muzzio, M. Tjahjadi, and J. M. Ottino, Phys. Rev. Let., 67, 54 (1991). 